\title{
STUDIES OF MAGNETIC CORES FOR JHF SYNCHROTRONS
}

\author{
M.Fujieda*, Y.Mori, H.Nakayama, C.Ohmori, S.Sawada, and Y.Tanabe ${ }^{\dagger}$, \\ KEK-Tanashi, 3-2-1 Midori-cho, Tanashi 188, JAPAN \\ E.Ezura, A.Takagi, M.Toda and M.Yoshii, KEK, 1-1 Oho, Tsukuba 305, JAPAN \\ T.Tanabe and T.Uesugi, CNS, University of Tokyo, 3-2-1 Midori-cho, Tanashi 188, JAPAN
}

\begin{abstract}
Magnetic cores suited for the RF cavities of JHF (Japanese Hadron Facility) synchrotrons have been studied. The shunt impedance of the cavity is required about $2 \mathrm{k} \Omega / \mathrm{m}$ because of the high acceleration voltage of more than $10 \mathrm{kV} / \mathrm{m}$. The permeability of the core should meet with the RF frequencies of the $3 \mathrm{GeV}$ booster and the $50 \mathrm{GeV}$ ring. Characteristics of several ferrites and a Fe-based soft magnetic alloy composed of amorphous and ultra-fine grain structure have been measured with a test bench. The shunt impedance of the soft magnetic core named "FINEMET" is high enough. It is stable when input RF power is increased, while those of samples of ferrites drop rapidly. The permeability of "FINEMET" strongly depends on the bias current. Because of its low $Q$ value, the bias current is not necessary for its new material. "FINEMET" is considered as one of the most probable candidate for JHF synchrotrons.
\end{abstract}

\section{INTRODUCTION}

The main parameters of the two synchrotrons for the JHF (Japanese Hadron Facility) are given in Table 1.

Table 1 : Main parameters

\begin{tabular}{|l|c|c|}
\hline & $3 \mathrm{GeV}$ booster & $50 \mathrm{GeV}$ ring \\
\hline $\begin{array}{l}\text { average } \\
\text { beam current }\end{array}$ & $200 \mu \mathrm{A}$ & $9.6 \mu \mathrm{A}$ \\
average & & \\
circulating current & $7 \mathrm{~A}$ & $7 \mathrm{~A}$ \\
repetition rate & $25 \mathrm{or} 50 \mathrm{~Hz}$ & $0.3 \mathrm{~Hz}$ \\
accelerating voltage & $420 \mathrm{kV}$ & $270 \mathrm{kV}$ \\
RF frequency & $2 \sim 3.4 \mathrm{MHz}$ & $3.4 \sim 3.5 \mathrm{MHz}$ \\
\hline
\end{tabular}

Because of the space limit for the RF apparatus, we need the voltage of more than $10 \mathrm{kV} / \mathrm{m}$. For stable acceleration under high beam current, the shunt impedance seen by beam of more than $1 \mathrm{k} \Omega / \mathrm{m}$ is necessary. Taking into account the RF amplifier, we need more than $2 \mathrm{k} \Omega / \mathrm{m}$ for the real shunt impedance of the cavity. If about 20 cores can be loaded in one meter, we require maximum accelerating voltage more than $500 \mathrm{~V}$ and shunt impedance more than $100 \Omega$ per one core consequently.

In order to shift RF frequency tuned to momentum of proton, bias current is supplied. The permeability of core

\footnotetext{
* also : Nuclear Science Research Facility,Institute for Chemical Research, Kyoto University, Gokanosho, Uji, Kyoto 611, JAPAN

$\dagger$ also : TOSHIBA Co.
}

has to vary according to bias current quickly.

Consequently the required characteristics of a core are shown as follows:

- shunt impedance $\sim 100 \Omega$

- quick response for bias current

The measurements for searching cores satisfying above requirements was performed. This paper presents the results of their RF characteristics for 4 ferrites and one soft magnetic core, particularly about their permeability and shunt impedance.

\section{MEASUREMENT APPARATUS}

\subsection{Components of the test bench}

The measurements every one core were done with the test bench. The cross section of the test bench is shown in Figure 1.

The inner diameter of cavity was $732 \mathrm{~mm}$ and its height was $255 \mathrm{~mm}$. The magnetic core was put on between two cooling plates. It was held by a stainless steel plate and adhered to the cooling plates. The RF power was supplied through the RF feeder and flowed to the earth through the variable vacuum capacitors and inside of the cavity. The bias current up to $1000 \mathrm{~A}$ was able to be supplied.

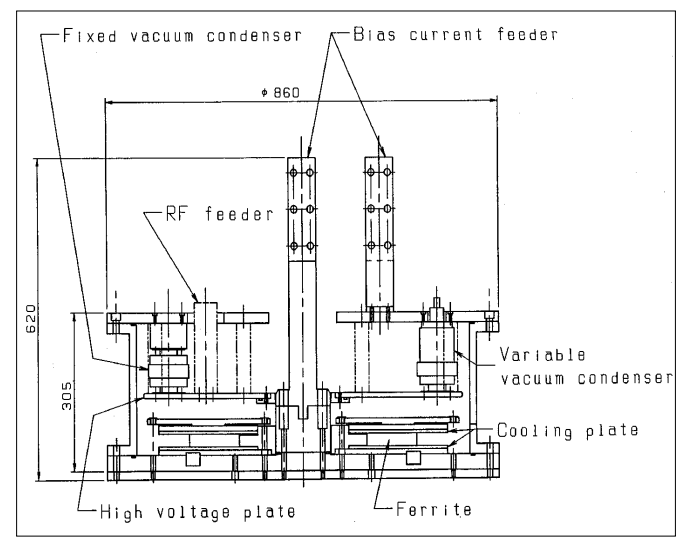

Figure 1: Cross section of the test bench. 


\subsection{Equivalent circuit}

Figure 2 shows the equivalent circuit including the RF amplifier and the bias current supply. The output impedance of the RF amplifier was $50 \Omega$. But the shunt impedance of the cavity was not usually $50 \Omega$. So the impedance matching circuit was incorporated between cavity and power supply.

The waveforms of the voltage and current detected by a probe and a current transformer were monitored by an oscilloscope. Then their maximum value $V_{\max }, I_{\max }$ and phase difference were measured and the impedance was calculated. The inductance was obtained from the resonant frequency and the value of the variable capacitors. The complex permeability and quality factor of the core were evaluated from the impedance and inductance.

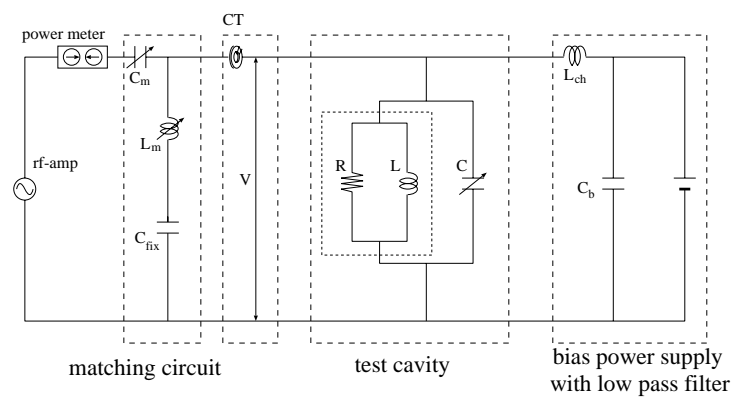

Figure 2: Equivalent circuit of the test bench.

\section{MEASUREMENTS}

\subsection{Magnetic cores}

We show the list of cores selected in Table 2.

Table 2: List of cores

\begin{tabular}{|l|c|c|c|}
\hline Name & I.D.[mm] & O.D. $[\mathrm{mm}]$ & $\mu_{p}^{\prime}$ at $3 \mathrm{MHz}$ \\
\hline \hline N5C & 260.0 & 635.0 & 225 \\
\hline FT3M & 320.0 & 579.0 & 2821 \\
\hline 4M2-302 & 200.0 & 500.0 & 39.5 \\
\hline 4M2-303 & 250.0 & 500.0 & 58.1 \\
\hline SY2 & 299.8 & 500.1 & 181 \\
\hline
\end{tabular}

I.D. and O.D. mean inner and outer diameter, respectively. The real permeability $\mu_{p}^{\prime}$ at $3 \mathrm{MHz}$ is shown in the third column of Table 2 . All the cores have one inch thickness. The cores except FT3M are Ni-Zn ferrites. N5C and SY2 have lower Q value and 4M2-302 and 4M2-303 have higher one than usual ferrites. FT3M is one of the soft magnetic cores named "FINEMET". This is a Fe-based soft magnetic alloy composed of amorphous and ultra-fine grain. This complex permeability is about $\left(\mu_{p}^{\prime}, \mu_{p}^{\prime \prime}\right)=$ $(1000,1000)$ around $4 \mathrm{MHz}$ and the Curie temperature is $570^{\circ} \mathrm{C}$.

\section{2 permeability}

The bias current dependence of the permeability and shunt impedance were measured. We fixed RF frequency at $3.0 \mathrm{MHz}$ and changed dc bias current from $0 \mathrm{~A}$ to $1000 \mathrm{~A}$. With changing the value of variable capacitors, the resonant condition was achieved. Figure 3 shows the bias current dependence of SY2 and FT3M. In the $3 \mathrm{GeV}$ booster, the RF frequency has to vary from $2 \mathrm{MHz}$ to $3.4 \mathrm{MHz}$. The permeability of All the samples varied enough within bias current of 1000A FT3M was more sensitive for bias current than SY2. We do not have to supply bias current because the Q value of FT3M is so low that FT3M keeps high impedance over the wide frequency range.

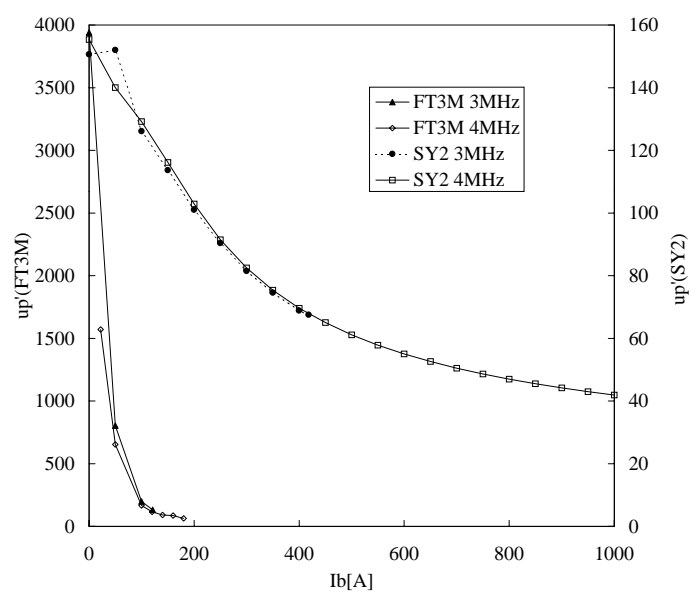

Figure 3: Bias current dependence of SY2 and FT3M.

\subsection{Shunt impedance}

Shunt impedance is given from the following formula.

$$
R_{p}=\mu_{0} t \ln \left(\frac{O . D .}{I . D .}\right) \times \mu_{p}^{\prime} Q f
$$

In this paper, $\mu_{p}^{\prime} Q f$ which is independent of size of core shows shunt impedance. $B_{R F}$ stands for RF flux density and it is written as : $B_{R F}=\frac{V_{R F}}{\omega S} . V_{R F}$ and $\mathrm{S}$ mean input $\mathrm{RF}$ voltage and cut surface area, respectively. If the accelerating voltage of $500 \mathrm{~V}$ per one core is replaced with $B_{R F}$, it becomes about $80 \sim 100$ gauss at $2 \mathrm{MHz}$. The increment of accelerating voltage as 2 times in the future is considered. Therefore the range of $B_{R F}$ applied for a core becomes about from 80 to 200 gauss. The shunt impedance and the $\mathrm{Q}$ value of ferrite are decreasing as the input RF power is increased. The temperature rise of core causes this and leads to reduce its shunt impedance. We have to apply more $\mathrm{RF}$ power to keep a constant voltage consequently. The core is desired to maintain a regular impedance for RF flux density. Figure 4 shows the change in shunt impedance for 
increase of RF flux density with dc bias current at $2 \mathrm{MHz}$. The data of $4 \mathrm{M} 2$ was measured at $2.7 \mathrm{MHz}$.

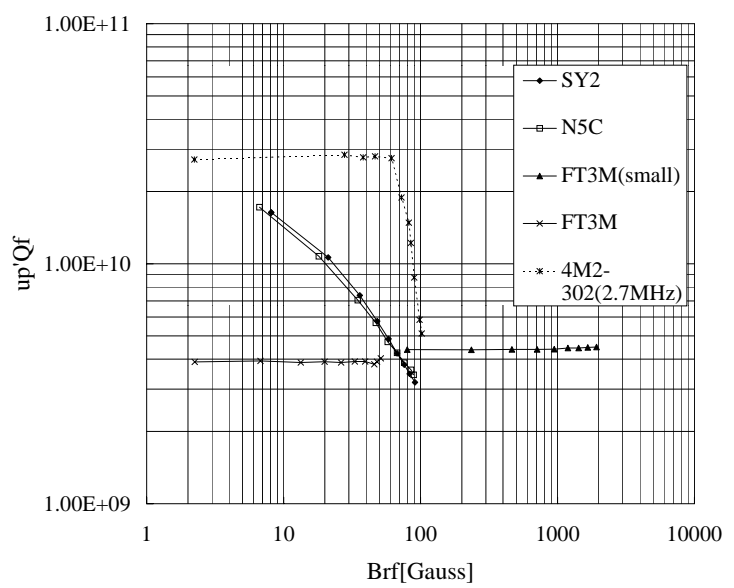

Figure 4: Change in shunt impedance for RF flux density.

Here the inner and outer diameter of FT3M(small) were $32 \mathrm{~mm}$ and $70 \mathrm{~mm}$, respectively. $4 \mathrm{M} 2$ had the highest shunt impedance. However it caused High Loss Effect(HLE) beyond about 60 gauss. Its shunt impedance was decreased suddenly, while FT3M kept stable impedance for $B_{R F}$. The ferrites except $4 \mathrm{M} 2$ had much higher impedance than FT3M up to about 70 gauss. But FT3M showed higher impedance in the practical range of $B_{R F}$.

In a proton synchrotron, RF frequency must be tuned as the revolution frequency of protons. Bias current is varied as rapid as the repetition rate of accelerator in order to shift RF frequency. The fast repetition occurs that the RF characteristics of magnetic cores become worse. So the test with varying ac bias frequency was performed. The result at the RF frequency of $2 \mathrm{MHz}$ and bias frequency of $50 \mathrm{~Hz}$ are shown in Figure 5. All ferrites had less shunt impedance than the results at the constant bias current.

\subsection{Others}

Temperature rise of FT3M was measured. Surface temperatures of FT3M were monitored with thermocouples. The temperature reached $140^{\circ} \mathrm{C}$ after 8 hours with RF power of $500 \mathrm{~W}$. FT3M did not show any variation of the characteristics.

FT3M has much higher Curie temperature than ferrites, as $570^{\circ} \mathrm{C}$. The cooling system of cores will be easy accordingly.

The RF cavity with "FINEMET" core will have low Q value. If the $\mathrm{Q}$ value of $\mathrm{RF}$ cavity is enough low to make beam instability dumped quickly, instabilities do not matter for high intensity proton synchrotrons.

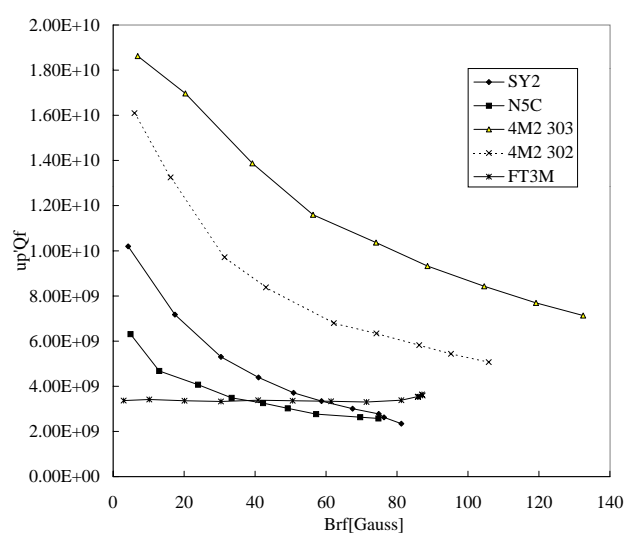

Figure 5: Shunt impedance for RF flux density with ac bias frequency of $50 \mathrm{~Hz}$.

\section{CONCLUSION AND PERSPECTIVE}

We studied characteristics of magnetic cores for JHF. The frequency and bias current dependences were measured. FT3M satisfies our required terms for both synchrotrons. The shunt impedance of FT3M is independent of RF flux density and very stable. It is not necessary for FT3M to be supplied bias current, for the Q value of FT3M is low enough. $4 \mathrm{M} 2$ seems to be suitable to $3 \mathrm{GeV}$ booster, but it cannot be used for $50 \mathrm{GeV}$ ring because of HLE.

Taking account of present results, we have developed a test RF cavity with FT3M cores. FT3M has very low Q value. The beam instability may be reduced by inserting FT3M to a RF cavity accordingly. But it is unknown how the beam loading is in case that the $\mathrm{Q}$ value is less than 1.0. The power test, temperature test and impedance measurement have been done for this cavity. The beam loading will be studied with electron gun and this cavity.

\section{REFERENCES}

[1] Y.Mori et al., INS Report, JHP-30 July(1996) (in Japanese).

[2] T.Uesugi et al., INS Report, JHP-31 January(1997) (in Japanese)

[3] K.Saito et al., Beam Science and Technology, published by Nuclear Science Research Facility, Inst. for Chem. Res., Kyoto Univ. 2(1997) pp.15-19. 